\title{
The Combination of Bacillus Subtilis wt55 with AiiO- Al06 Improve The Resistance of Zebrafish To $A$. Veronii Infection by Simple Collocation Rather Than B. Subtilis Quorum-Quenching Recombinant Expression Strain
}

\section{Yuan-Yuan Yao}

Institute of feed research, Chinese Academy of Agricultural Sciences

Rui Xia

Institute of Feed Research, Chinese Academy of Agricultural Sciences

Ya-Lin Yang

Institute of feed Research, Chinese Academy of Agricultural Sciences

Chen-Chen Gao

Institute of Feed Research, Chinese Academy of Agricultural Sciences

Feng-Li Zhang

Institute of Feed Research, Chinese Academy of Agricultural Sciences

Juan $\mathrm{Hu}$

Institute of Feed Research, Chinese Academy of Agricultural Sciences

Qiang Hao

Institute of Feed Research, Chinese Academy of Agricultural Sciences

Chao Ran

Institute of Feed Research, Chinese Academy of Agricultural Sciences

Zhen Zhang

Institute of Feed Research, Chinese Academy of Agricultural Sciences

Zhigang Zhou ( $\nabla$ zhouzhigang03@caas.cn )

Chinese Academy of Agricultural Sciences Feed Research Institute

Original article

Keywords: B. subtilis, quenching enzyme AiiO-AIO6, intestinal microflora, immune regulation, germ-free zebrafish

Posted Date: July 7th, 2021

DOl: https://doi.org/10.21203/rs.3.rs-502037/v2 
License: (c) (i) This work is licensed under a Creative Commons Attribution 4.0 International License. Read Full License 


\section{Abstract}

Disease problems will seriously restrict the sustainable development of aquaculture, and the environmental-friendly prevention strategies are urgently needed. Probiotics and quorum-quenching enzyme are innovative strategies to control bacterial diseases. The bacteriostatic effect of $B$. subtilis wt55 strain and quenching enzyme AiiO-AlO6 in vitro showed wt55 inhibit the growth of $A$. veronii, but AiiO-AlO6 did not. Therefore simple combination of B. subtilis wt55 strain and AiiO-AlO6 was used to evaluate potential synergistic effect. The results showed this combination could significantly reduce the number of invasive $A$. veronii in gut after challenge, corresponding to the lower intestinal alkaline phosphatase activity. In vitro co-culture experiments showed this combination could inhibit the growth of A. veronii. Direct immersion of germ-free zebrafish proved AiiO-AlO6 did not directly regulate the innate immune response of the host, but wt55 did it, and the combination of wt55 and AiiO-AlO6 could significantly reduce the expression of NF-KB and proinflammatory cytokine IL- $1 \beta$, increase the expression of lysozyme gene. The gut microbiota induced by either experimental diet was transferred to germ-free zebrafish, and the results showed that intestinal microbiota also plays a regulatory role. The gut microbiota from combination group could significantly inhibit the expression of IL-1 $\beta$ and NF-KB, and increased the expression of TGF- $\beta$ and lysozyme. Given the effectiveness of this combination, $a B$. subtilis quorum-quenching recombinant expression strain in which AiiO-Al06 was surface displayed on the spores and secreted by vegetative cells was built. The results showed that the survival rate after challenge was lower than that of the group treated with AiiO-AlO6 or wt55 alone, and the expression of proinflammatory cytokine IL-1 $\beta$ and NF-kB were significantly higher. Our study demonstrated the effectiveness of $B$. subtilis and AiiO-AIO6 simple combination and established an efficient $B$. subtilis expression system.

\section{Introduction}

The development of aquaculture in China is booming, China alone supplied $58 \%$ and $59 \%$ of the global aquaculture volume and value, respectively, for all categories combined in 2017 (Naylor et al. 2021). China's aquatic industry occupy a decisive position in the world, the new fisheries and aquaculture reforms and policies to be implemented by China are expected to have a noticeable impact at the world level (FAO 2020). In China, intensive and industrial aquaculture is the development trend at now and in the next decade (FAO 2020), accompanied by is the increasingly prominent disease problems. It is mainly reflected in the variety of pathogens, high incidence, rapid transmission, wide range and high fatality rate, among which bacterial diseases is an important impediment to aquaculture because bacteria can survive well in aquatic environments independently of their hosts (Gui and Zhang 2018). As a result, the abuse of antibiotics and chemical agents and their derived side effects such as drug residues, food hygiene and safety, environmental pollution and other problems have been paid more and more attention, research and development of green inputs alternatives are urgent (Lieke et al. 2019).

Probiotics, when administered in adequate amounts, have the function to improve the physiology, growth performance, and immune responses of host including aquaculture-related species. Now probiotics as an 
eco-friendly feed additive, is wildly applied in aquaculture at the farm level to enhance the economic performance (Subedi and Shrestha 2020). Various aquatic probiotics have been reported which show activity not only against bacterial pathogen, but also against fungus and virus (Chauhan and Singh 2019). The sporulation capacity and the production of antimicrobial substances and enzymes provide species of Bacillus with the ability to colonize in different habitats and to contribute benefit for host health, and they have been widely used as environmental and dietary probiotics in aquaculture (Soltani et al. 2019). Bacillus subtilis, which is approved use by the Chinese Ministry of Agriculture, is one of such probiotics that is very well-researched and most widely used in aquaculture, and no adverse consequences have been reported till date (Nayak 2021). Moreover, B. subtilis has been studied for decades as an excellent host to express heterologous proteins (Wong 1995).

Based on the basic research of bacterial diseases, it is found that quorum sensing (QS) among bacteria is very important for their physiological function. For the same species of bacteria, bioluminescence, biofilm formation, secretion of extracellular polymeric substances, immune response and other biological characteristics of bacteria can be regulated through the communication of QS signal molecules, thereby affecting their virulence and pathogenicity (Eickhoff and Bassler 2018; Høyland-Kroghsbo et al. 2017). For different species of bacteria, bacterial QS can shape the composition of microbial communities (Mukherjee and Bassler 2019; Papenfort and Bassler 2016). For infected hosts, bacterial QS signal molecules also regulate the immune response and disease resistance of hosts (Huang et al. 2018; Kariminik et al. 2017). Therefore, by interfering with the QS system between microbial cells, the quorumquenching (QQ) strategy becomes a new way to prevent and control the infection of pathogenic bacteria (Jiang et al. 2019). For QQ, there are mainly two ways: competitive inhibition (Kalia et al. 2019) and signal molecules degradation (Bzdrenga et al. 2017). Because the QQ enzyme degrades signal molecules is an extracellular catalytic reaction, it is currently a more effective and least toxic way of QQ (Guendouze et al. 2017). AiiO-AlO6 is a lactonase, it works via enzymatic inactivation of $N$-acylhomoserine lactones (AHLs), the signal molecules of Gram-negative $\left(\mathrm{G}^{-}\right)$bacteria. We have previously studied its enzymatic characteristics, prokaryotic expression and its effect on A. hydrophila virulence factors (Pan et al. 2011). However, AiiO-AIO6 is not always effective in practical application due to the complex aquatic environment, that include various types of pathogens which not just using AHLs as signal molecules and viruses. Therefore, strategies to improve the effectiveness of QQ enzyme are needed, and probiotics used in conjunction with QQ enzyme may complement and increase its effectiveness.

A wild $B$. subtilis named wt55 strain was isolated from the mucus of sturgeon in our laboratory. After evaluating the effects of wt55 and AiiO-AlO6 on the growth of $A$. veronii, this study is intended to explore the strategy about the combinatory use of $B$. subtilis wt55 and AiiO-AlO6 to stabilize or improve disease resistance of host. Taking zebrafish as the model, this study firstly evaluated simple combination of wt55 and AiiO-AlO6 (wt55 + AlO6). Then a simpler compatibility strategy namely B. subtilis QQ recombinant strain wt55-AlO6 in which AiiO-AlO6 was surface displayed on the spores and constitutive secretory expression by vegetative cells was constructed and evaluated. This study will lay a foundation for the efficient use of probiotics and QQ enzymes and may provide technical support for the green disease 
prevention and control in aquaculture. Moreover, an efficient $B$. subtilis expression system was established, which provided a basis for the expression and application of other proteins in the future.

\section{Materials And Methods}

\section{Fish and feeding conditions}

Two-month-old zebrafish with similar weight $(0.082 \mathrm{~g})$ were randomly dispersed into four tanks for each group $(26.5 \times 14.5 \times 18 \mathrm{~cm})$ at a density of 20 fish after acclimated two weeks, all of which were cultured in the recirculating water system (at $60 \mathrm{~L} / \mathrm{h}$ ). During the feeding period, the fish were given an experimental diet twice a day (9:00 and 17:00), the temperature was maintained at approximately $28^{\circ} \mathrm{C}$, the dissolved oxygen was $>6.0 \mathrm{mg} / \mathrm{L}$, the $\mathrm{pH}$ was $7.0-7.2$, the nitrite content was $<0.005 \mathrm{mg} / \mathrm{L}$, and the ammonia nitrogen was $<0.2 \mathrm{mg} / \mathrm{L}$.

\section{Bacterial strains and growth conditions}

B. subtilis wt55 strains isolated from sturgeon and kept in China General Microbiological Culture Collection Center (CGMCC No. 1.19039) were directed at using in combination with AiiO-AIO6 and constructing recombinant strains which could display QQ enzyme AiiO-AlO6 on the surface of spores and secrete this protein by vegetative cells. Proliferation of $B$. subtilis were routinely in Luria-Bertani (LB) broth at $37^{\circ} \mathrm{C}$, and the spores were produced in Difco-Sporulation medium (DSM) using the exhaustion method. Construction, transformation, screening and sequencing of recombinant plasmid were performed in Escherichia coli strain DH5a, it grows well in LB. The pathogenic bacteria A. veronii Hm091 also kept in China General Microbiological Culture Collection Center (CGMCC No. 22536) could be cultured in LB and has ampicillin resistant through identify. This pathogen was used to infect zebrafish and determine the disease resistance of the host, also used for co-culture with B. subtilis wt55 or AiiOAlO6 to test the antimicrobial properties of them.

\section{Construction and confirmation of recombinant expression $B$. subtilis strain}

A recombinant $B$. subtilis strain expressing AiiO-AlO6 protein on the surface of $B$. subtilis spore and constitutive secretion of vegetative cells was constructed, the construction diagram was shown in Fig. 2. The anchoring protein selected for spore surface display was CotC, and the gene of AiiO-AlO6 was linked to its $\mathrm{N}$ terminal. The target genes by gene synthetic are respectively BamH I cutting site, CotC promoter, His tag, AiiO-AIO6, linker with alpha-helix structure, CotC and EcoR I cutting site, which string together in sequence. This gene segment was digested by BamH I and EcoR I and then inserted into the vector pDG364 which go through the same double digestion. The resulted recombinant plasmid named pDG364-N-AIO6 was transferred into the wild strain of $B$. subtilis wt55 by chemical transformation and homologous recombination was carried out at the homologous arm (amylase gene). Chloramphenicol resistance of $10 \mu \mathrm{g} / \mathrm{mL}$ was used to screen the positive clones. Chromosomal integration of exogenous gene was verified using amylase activity and genomic PCR (primers are listed in Table 1). After inducing 
spore generation, the AiiO-Al06 displayed on the surface of wt55 spore was confirmed through western blot (WB) and immunofluorescence microscopy. The positive clone was named wt55-N-AIO6(SD).

The plasmid pWB-AIO6BS which could secrete AiiO-AIO6 in B. subtilis was constructed in our laboratory before (Pan et al. 2016), and the extracted plasmid was convert to transform competent cells of wt55- $\mathrm{N}$ AI06(SD) strain by chemical method. The concentration of kanamycin to screen positive clones was 25 $\mu \mathrm{g} / \mathrm{mL}$, and the clones were verified by plasmid extraction and WB of secreted AiiO-AIO6 in supernatant. The enzyme activity on spore surface and secretion in supernatant were determined by high performance liquid chromatography (HPLC) according to the method previously reported (Xia et al. 2020). The final bifunctional recombinant strain was named wt55-AlO6. The information of plasmids and strains used in this paper are shown in Table 3.

\section{Diets and rearing experiments}

Feeding trial firstly evaluated the effect of simple combination of B. subtilis and AiiO-AlO6. Four experimental diets were prepared: basal diet (ck), diets with each gram of feed contains $10 \mathrm{U}$ QQ enzyme AiiO-AlO6 (AlO6), AiiO-AlO6 with the same enzyme activity paired with $B$. subtilis wt55 $\left(10^{8} \mathrm{cfu} / \mathrm{g}\right.$ feed, wt55+Al06) or the same number of wt55 strain (wt55). Protein and bacteria were added directly during feed preparation, the feed was air-dried at room temperature and homogenized with a grinder. Fish were fed $6 \%$ of body weight per day, increase by $1 \%$ after a week, turn off the circulating water during feeding, and the experiment lasted for two weeks.

Another evaluation research about the recombinant wt55-AlO6 strain on the disease resistance of the host was carried out. The experiment was divided into four groups, fish were respectively fed with basal diet as control (ck), diets supplemented with AiiO-Al06 which enzyme activity was $5 \mathrm{U} / \mathrm{g}$ feed (AlO6), recombinant spores with the same enzyme activity (about $2 \times 10^{9} \mathrm{cfu} / \mathrm{g}$ feed, wt55-AlO6) or the same number of $B$. subtilis wt55 strain (wt55). Feed preparation, breeding method and time were the same as above.

\section{Sampling and Quantitative PCR testing}

After the end of the breeding experiment, all fish were starved for $24 \mathrm{~h}$ before sampling. Intestines were mainly taken, samples from 2-3 fish were randomly taken and mixed into the one. As for zebrafish larvae, about 15 fish would be used as a sample, and each group all had six replicates. Total RNA was extracted by TRIZOL Reagent (Invitrogen) after homogenate, cDNA was synthesized using fastKing gDNA dispelling RT superMix (TianGen) to eliminate contaminated genomic DNA. RPS11 was used as reference gene for the expression of all detected genes, quantitative real-time PCR ( $q$ PCR) reaction was performed using the SYBR Green Supermix (TianGen) on the Light Cycler 480 (Roche 480). The final results show was relative mRNA expression compared to the value of the ck control group. The primers for each gene were given in Table 1.

\section{Co-culture of $\boldsymbol{A}$. veronii Hm091 in vitro}


In order to investigate the effect of $B$. subtilis wt55, AiiO-AlO6 or wt55 combination with Al06 on the growth of $A$. veronii $\mathrm{Hm091}$, mixed culture on liquid medium was done. A. veronii and wt55 were separately cultured overnight, and OD600 was adjusted to be the same before transfer. Four culture groups were set up: $\mathrm{PBS}+A$. veronii as control, AiiO-AlO6 + A. veronii, wt55+AlO6 + A. veronii and wt55 $+A$. veronii. Then $100 \mu \mathrm{L}$ of overnight cultured bacteria or sterile enzyme fluid were inoculated in $100 \mathrm{~mL}$ LB medium, each culture group has three flask replicates. The inoculated flasks were incubated at $37{ }^{\circ} \mathrm{C}$ with shaking at $200 \mathrm{rpms}$ in an orbital incubator. At $4,8,12,24 \mathrm{~h}, 1 \mathrm{~mL}$ of cultures were sampled to do gradient dilution and count the number of $A$. veronii. Because of the different colony morphology, $A$. veronii and $B$. subtilis could be distinguished during plate counting.

\section{Gut alkaline phosphatase activity and $A$. veronii counts in gut after challenge}

Multiple clones of $A$. veronii on the plate were selected into $1 \mathrm{~mL} \mathrm{LB}$ to make their OD $600=0.45$, then transferred $200 \mu \mathrm{L}$ to $60 \mathrm{~mL}$ fresh LB medium and cultured for $18 \mathrm{~h}$ at $37^{\circ} \mathrm{C}$ with shaking at $200 \mathrm{rpms}$. Thirty fish from each group were randomly assigned to three tanks, $A$. veronii with final concentration of about $5 \times 10^{8} \mathrm{cfu} / \mathrm{mL}$ were added to each tank, fish were fasting during pathogen infection. Three fish from each tank were chosen for detecting intestinal alkaline phosphatase (IAP) $24 \mathrm{~h}$ post challenge using Alkaline Phosphatase Assay Kit (Beyotime). Separate intact intestines were homogenized in sterile PBS and then applied on a plate containing ampicillin to count the number of $A$. veronii which infected the gut, counts from unchallenged fish were used as background values.

\section{Germ free zebrafish production and experimental treatment}

Germ free (GF) zebrafish were produced according to reported protocols (He et al. 2017; Oyarbide et al. 2015). At the third days post fertilization (dpf), sterility was tested, after that $80 \%$ gnotobiotic zebrafish media (GZM) was changed, the egg membranes and unhatched embryos were sucked away.

GF-zebrafish were treated on $5 \mathrm{dpf}$, and two experiments were carried out. Experiment 1 was about sterile AiiO-AlO6 (final concentration $10 \mathrm{U} / \mathrm{mL})$, collocation of $B$. subtilis wt55 $\left(10^{6} \mathrm{cfu} / \mathrm{mL}\right)$ with AiiO-AlO6 $(10$ $\mathrm{U} / \mathrm{mL})$ or wt55 $\left(10^{6} \mathrm{cfu} / \mathrm{mL}\right)$ directly immersed GF-zebrafish, the untreated group served as control. Experiment 2 was gut microbes from four groups of rearing experiments (ck, AlO6, wt55+AlO6 and wt55) to transfer GF-zebrafish. Simply, intestinal contents of five fish were sampled $4 \mathrm{~h}$ after the last feeding at the end of the breeding experiment. After fully mixed, the impurities were removed by low-speed centrifugation and the bacteria with the final concentration of $10^{6} \mathrm{cfu} / \mathrm{mL}$ were added to the GF-zebrafish culture flask which with the same fixed volume of GZM $(30 \mathrm{~mL})$. Every group had six replicates, and zebrafish larvae were collected after three days of immersion treatment.

\section{Statistical analysis}

Data were analyzed by one way analysis of variance (ANOVA) with Duncan's post hoc test. Data figures were made in GraphPad Prism Version 8 software. In all cases, differences were considered statistically significant when $p<0.05$. 


\section{Results}

Effects of B. subtilis wt55 and AiiO-AlO6 on the growth of A. veronii

Firstly, the effects of single use of $B$. subtilis and AiiO-AlO6 on growth of $A$. veronii in vitro were evaluated. A. veronii was cocultured with AiiO-AlO6 or wt55, the results showed that QQ enzymes AiiO-AlO6 did not have any negative effect on growth of $A$. veronii, because the bacterial count though fluctuated a little compared to the control group, but there was no significant difference. The antagonistic activity of $B$. subtilis against $A$. veronii was very significant at the early growth stage (Table 2 ). In view of the different effects on pathogenic bacteria, the strategy of using $B$. subtilis to match AiiO-AlO6 to further improve its effectiveness has been studied.

\section{Combination of wt55 and AiiO-Al06 improve the resistance of zebrafish to A. veronii by growth inhibition, direct immune regulation and indirect mediation of intestinal microbiota}

The efficacy about a simple combinatory use of wt55 with AiiO-AlO6 (wt55 + AlO6) was evaluated. Most of all, the disease resistance of zebrafish was assessed. The count of pathogenic bacteria in each group after challenge showed that the burden of $A$. veronii in the group supplemented with AiiO-AlO6 or wt55 were significantly lower when compared with control group, and zebrafish treated with wt55 + AlO6 exhibited the lowest Hm091 counts in the gut, suggesting this strategy could inhibit the binding and/or proliferation of $A$. veronii more efficiently (Fig. 1a). This was consistent with the lower IAP activity post challenge in experimental group (Fig. 1b).

Furthermore, the mode of action behind the effectiveness of this combination strategy was investigated. In vitro, the effect on $A$. veronii growth was first evaluated. The result showed that addition of wt55+AlO6 significantly reduced the amount of $A$. veronii at the early growth stage, and the antagonistic property of B. subtilis was the main cause of this effect (Table 2).

In vivo, directly or/and mediated by gut microbes to indirectly regulating the immune response of host was assessed in GF zebrafish. For directly immersed GF-zebrafish, there were no significant changes in expression of inflammation related gene (NF-KB, IL-1 $\beta$ and TGF- $\beta$ ) and lysozyme gene in GF zebrafish when treated with AiiO-Al06. In wt55 + AlO6 group, relative expression of NF-kB was decreased significantly, transcription of IL-1 $\beta$ and TGF- $\beta$ did not change compare with control, while the expression of lysozyme gene was significantly increased. As for GF-zebrafish treated with wt55, the inflammationrelated responses were significantly changed, as well as lysozyme (Fig. 1C). For GF-zebrafish transferred by gut microbiota taken from diet supplemented with either AiiO-AlO6, wt55 + AlO6 or wt55 or the control diet, as show in Fig. 1d, compared to control treatment, gut microbiota from AiiO-AlO6 did not affect the gene expression of NF-KB, IL-1 $\beta$ and lysozyme gene, expression of anti-inflammatory TGF- $\beta$ was significantly increased. The profile of NF-KB and IL-1 $\beta$ expression induced by gut microbiota of wt55+ AlO6 was decreased significantly, TGF- $\beta$ expression had no significant change and expression of lysozyme gene was significantly up-regulated. In microbiota of wt55 treatment group, the relative 
expression level of NF-KB was not changed, IL-1 $\beta$ was significantly down-regulated, but the expression of TGF- $\beta$ and lysozyme gene were significantly increased.

\section{Successful construction of the recombinant B. subtilis wt55-Al06 expression strain}

Because the simple combination worked well, a simpler and more practical way was built. Validation of recombinant wt55-AlO6 strain included surface display and secretory expression of AiiO-AlO6 was done. Firstly, chromosomal integration of plasmid pDG364-N-AIO6 was confirmed. Because homologous recombination occurred at the amylase gene, the amylase activity of wt55-Al06 was destroyed and lost its function to hydrolyze starch, so the starch around the recombinant strain turned blue when it encountered with iodine solution and no white halo appeared, while the wt55 strain produced a big white circle around the colony (Fig. 3a). The introduction of foreign genes was further validated by PCR, genomes of wt55 and recombinant wt55-Al06 strain were extracted, specific primers for AiiO-AlO6 and amylase were used. Because the wt55 did not have AiiO-Al06 gene, so it could not be amplified, however a fragment about $800 \mathrm{bp}$ could be amplified in recombinant strain. When using amylase gene as primer, 2000 bp was obtained in wt55, but in wt55-Al06 was about 4800 bp which included the elements for surface displaying of AiiO-AIO6 and resistance screening of clones in plasmid pDG364-N-AlO6 because of homologous insert (Fig. 3b). Therefore, the above results all proved that exogenous genes have been successfully integrated into the genome of $B$. subtilis.

Secondly, expression of AiiO-AlO6 on spores of wt55-Al06 strain was detected. Spore coat proteins were extracted to detect protein expression by WB using His-tag antibody. As show in Fig. 3d, wt55 did not have AlO6-specific bands at the protein level, while the wt55-AIO6 could be detected a clear band of a suitable size, about $40 \mathrm{kD}$, which was exactly the size of the fusion expression of AiiO-AlO6 and its anchor protein CotC. Similarly, immunofluorescence microscopy detection showed green fluorescence signal only appeared on the surface of recombinant spores (Fig. 3c). Therefore, the above results indicated that AiiO-AlO6 was successfully expressed on the surface of wt55-Al06 spores.

Next, constitutive secretion of the AiiO-AlO6 protein in wt55-Al06 was validated. The secreted expression plasmid pWB-AIO6BS could be successfully extracted from the recombinant strain, and the cells of wt55 had no plasmid (Fig. 3b). B. subtilis was cultured in LB medium for $24 \mathrm{~h}$ and then supernatant was concentrated to conduct WB, the target band suitable for Al06 was only detected in wt55-AlO6 (Fig. 3d). These results indicated that the recombinant strains of wt55-Al06 successfully expressed and secreted AiiO-AlO6 in their vegetative cells stage.

Final was the detection of wt55-AIO6 specific activity to degrade signal molecule substrate 3-oxo-C8-HSL. By quantification, the enzyme activity of wt55-Al06 spores was about $320 \mathrm{U} /$ one hundred billion cells, the enzyme activity of secreted AiiO-AlO6 was about $146 \mathrm{U} / \mathrm{mL}$.

Recombinant B. subtilis wt55-Al06 expression strain was not as efficient as simple combination on disease resistance of zebrafish 
Then its effects on disease resistance of zebrafish were evaluated in vivo. Four groups of zebrafish fed with AiiO-Al06, wt55-Al06 spores or with the wt55 native spores or basal diet were challenged by $A$. veronii. According to the survival curve (Fig. 4a), compared with the control group, additive of AiiO-AlO6 or wt55-Al06 or wt55 all improved the survival rate of zebrafish, but the recombinant strain did not show the expected advantage. Further study of inflammatory reaction in gut was conducted after feeding. For AlO6 group, the transcriptional levels of pro-inflammatory factor IL-1 $\beta$ did not change compare to control group, NF-KB even decreased significantly, anti-inflammatory cytokine IL-10 was significantly upregulated. In wt55-Al06 group, mRNA level of inflammatory cytokines (IL-1 $\beta$ and NF-KB) were increased significantly, while IL-10 has no obvious change. On the contrary, the gene expression of IL-1 $\beta$ and NF-KB in wt55 group did not show a significant change, while anti-inflammatory cytokine was significantly higher than that in the control group (Fig. 4b).

\section{Discussion}

The direct regulation of the additive on pathogens is very important to enhance the disease resistance of host. $B$. subtilis is a probiotics that could inhibit the growth of aquaculture pathogens such as Vibrio and Aeromona sps. (Nair et al. 2021), which is consistent with the results of this study. QQ enzyme AiiO-AlO6 has a biotherapeutic potential against pathogens of aquatic organisms, not by growth inhibition which is different from traditional antibiotic treatment and thus does not easily cause resistance (Haque et al. 2018), but by regulation of virulence gene expression as with other QQ enzymes (Defoirdt, 2018), and may also lead to increased sensitivity to antibiotics and phages (Mion et al. 2019). Probiotics and QQ enzymes work in different ways, in view of this, we assume that their combination will enhance the effect. Therefore, the combinatory use of wt55 and AiiO-AlO6 (wt55 + AlO6) was preliminarily evaluated. Since fish reside in the aquatic environment, mucosal surface, such as gills, skin, or gut are continuously exposed to pathogens, and become the major portal of entry (Yu et al. 2020). Infection with A. veronii $\mathrm{Hm} 091$ can cause intestinal damage, and into regions around the intestine and in the abdomen (Ran et al. 2018). The usage of AiiO-AIO6 or wt55 could significantly reduce the number of enteric pathogens after challenge, and the combination of wt55 and AiiO-Al06 had the best exclusion effect. LPS is a major component of the outer membrane of $A$. veronii, endotoxemia resulting from the abundance of LPS in the gut or blood stream can lead to inflammatory response (Novoa et al. 2009) even subsequent complications and death. IAP plays a vital role in promoting mucosal tolerance to gut bacteria via dephosphorylation of LPS to attenuated LPS-mediated toxicity (Bilski et al. 2017), which was consistent with the results in this experiment, i.e., lower bacterial load was associated with lower IAP activity, and vice versa. In previous studies, fewer infective bacteria and lower IAP activity in the gut were associated with higher survival rates (He et al. 2017; Liu et al. 2016), therefore the result of this study can indirectly indicate that the combination of wt55 and AiiO-AlO6 has a good application effect.

Further, the mechanism behind the effectiveness of the wt55 + Al06 was explored. Firstly, its antagonistic

action against $A$. veronii in vitro was evaluated. The results showed that the wt55 + Al06 had an inhibitory effect on it, and this effect was mainly provided by $B$. subtilis. The potentiality of competitive exclusion of the $B$. subtilis may be the production of antimicrobial substances, which have antimicrobial effects 
against broad spectrum of pathogens (Caulier et al. 2019). These substances are natural part of human antimicrobial defense system hence the possibility of developing pathogen resistance or unwanted side effects is less (Suva et al. 2016). This may also be one reason why the number of invading $A$. veronii decreased in wt55 treatment group after challenge. In addition, $B$. subtilis can eliminate pathogens by competing with them for nutrients or adhesion sites (James et al. 2021). And B. subtilis spores which used as a form of administration may also be effective agents in the control of pathogens (La Ragione and Woodward, 2003).

Then, by using GF zebrafish model, the direct or indirect effect (mediated by intestinal flora) of wt55 + AlO6 to regulate fish immunity were studied. GF zebrafish model provides convenience for the study of the function of intestinal microorganisms and the interaction mechanism between additive and the host, and the establishment and application of this model have been skilled (Tan et al. 2019). The case of AiiOAl06 or B. subtilis wt55 directly immerse GF zebrafish showed AiiO-Al06 does not regulate the host immune response, but wt55 does, hence wt55 + Al06 can enhance defence against pathogens. It is widely accepted that $B$. subtilis can regulate the innate immune response of the fish (Galagarza et al. 2018; Olmos et al. 2020), but the way it works is unclear. This work provides the evidence that $B$. subtilis can directly regulate inflammatory response and lysozyme transcription of zebrafish larvae, suggesting that the immune-activating effect of $B$. subtilis is self-related. Previous studies also have shown that $B$. subtilis stimulated dendritic cells of grass carp, and several pro-inflammatory cytokines and antiinflammatory cytokines genes expressions are significantly increased (Zhou et al. 2019). In addition, the intestinal microbiota induced by dietary additive also has a positive effect on host immune response. The use of AiiO-AlO6 will interfere the QS signal molecules of some intestinal microbiota and alter gut microbial community structure. B. subtilis also can modulate bacterial flora in the gut (Shi et al. 2020), as well as spore supplementation (Jang et al. 2021). The symbiotic relationship and interaction between intestinal microbiota and host make each other in a dynamic equilibrium state, many studies have confirmed the positive role of intestinal microbiota in improving fish immunity (Teame et al. 2020; Wu et al. 2020a; Wu et al. 2020b), and our results are consistent with them. Together, these results showed that probiotic properties of $B$. subtilis enhance the effect of AiiO-AIO6.

The above results proved the effectiveness of the combination use of wt55 and AiiO-AlO6, for purpose of more convenient use and lay the foundation for the product formulation, a QQ recombinant strain wt55AlO6 which had the functions of both probiotics and QQ enzyme was constructed. Although many AHL lactases were derived from the genus of Bacillus, the expression of $B$. subtilis is rarely reported (Huma et al., 2011). Spore of $B$. subtilis is one of the excellent platforms for displaying exogenous proteins on its surface (Zhang et al. 2019). N-terminal fusion and linker with secondary structure were chosen in this study according to the spore surface display strategy, which contributes to a higher efficiency of display and stability of protein (Yao et al. 2020). AiiO-AlO6 is located on the surface of spores and can react directly with substrates. The unique stress resistance of spores endows the protein on spores with thermal stability, acid and alkali resistance, enzymatic hydrolysis resistance and other characteristics, and does not affect the biological activity of displayed proteins, so it is more suitable for feed processing industry. And when the spores of $B$. subtilis enter the intestinal tract of the host, some of them will 
germinate into vegetative cells (Latorre et al. 2014), constitutionally secreted expression during proliferation of $B$. subtilis was built as a supplement. The recombinant expression strains of wt55-AlO6 could ensures the long-term expression and efficacy of AiiO-AIO6. But the use of this recombinant strain in zebrafish caused a quicker death after challenge when compared with using AiiO-Al06 or wt55 alone. Further investigation revealed that an inflammatory response in gut of wt55-Al06 may be responsible for the unexpected results. In this study, the alone use of same dosage wt55 and AiiO-AlO6 did not cause inflammatory response, one possible difference between wt55-AIO6 and AiiO-AIO6 or wt55 was the enzyme activity in wt55-AlO6 group was not likely to be digested and degraded, but could be maintained at a constant level. Moreover, with daily feeding, the accumulation may be increased, and if some $B$. subtilis germinated and proliferated, it is difficult to estimate the actual amounts of enzymes at work. In the GF zebrafish model, AiiO-AlO6 did not directly regulate the host immune response, but the enzyme induced intestinal microbiota did. So we guess that changes in the microbiota mediated by AiiO-AlO6 may negatively affect the inflammatory response of the host. However, this specific mechanism needs to be further studied. The surface display and constitutive secretory expression of $B$. subtilis is a good expression system, which may be due to the properties of quenching enzyme AiiO-AlO6, and it should play a better role in industrial production or other types of protein applications.

\section{Abbreviations}

QQ

quorum quenching; GF:germ-free; QS:quorum sensing; LB:Luria-Bertani; DSM:Difco-Sporulation medium; qPCR:quantitative real-time PCR; HPLC:high performance liquid chromatography; WB:western blot; IAP:intestinal alkaline phosphatase; GZM:gnotobiotic zebrafish media; AHLs: $N$-acylhomoserine lactones; $\mathrm{G}^{-}$:Gram-negative.

\section{Declarations}

\section{Authors' contributions}

Participated in research design and performed data analysis: ZZ, YY, YY, RC and ZZ; conducted experiments and wrote the manuscript: YY; provided technical support: FZ, CG, RX, HJ and HQ. All authors read and approved the final manuscript.

\section{Funding}

This work was supported by grants from the National Natural Science Foundation of China (Grant Nos. 3180131599 and 31925038), the earmarked fund for Modern Agro-industry Technology Research System (SCGWZJ20211104-4), the Innovation Capability Support Program of Shaanxi (2018TD-021) and the National Key Basic Research Program of China (973 Program) (2015CB150605).

\section{Availability of data and materials}


All the data are presented in the main paper or the additional information.

\section{Ethics approval and consent to participate}

All experimental zebrafish in this study were conducted in agreement with protocols approved by the Institute of Feed Research of Chinese Academy of Agricultural Sciences Animal Care Committee, under the auspices of the China Council for Animal Care (AssuranceNo.2018-AF-FRI-CAAS-001).

\section{Consent for publication}

Not applicable.

\section{Competing interests}

The authors have no conflicting commercial or financial interest in publishing this paper.

\section{Acknowledgements}

Not applicable.

\section{References}

1. Bilski J, Mazur-Bialy A, Wojcik D, Zahradnik-Bilska J, Brzozowski B, Magierowski M, Mach T, Magierowska K, Brzozowski T (2017) The role of intestinal alkaline phosphatase in inflammatory disorders of gastrointestinal tract. Mediat Inflamm 2017:9074601

2. Bzdrenga J, Daude D, Remy B, Jacquet P, Plener L, Elias M, Chabriere E (2017) Biotechnological applications of quorum quenching enzymes. Chem-Biol Interact 267:104-115

3. Chauhan A, Singh R (2019) Probiotics in aquaculture: a promising emerging alternative approach. Symbiosis 77(2):99-113

4. Defoirdt T (2018) Quorum-sensing systems as targets for antivirulence therapy. Trends Microbiol 26(4):313-328

5. Eickhoff MJ, Bassler BL (2018) Snapshot: Bacterial quorum sensing. Cell 174(5):1328

6. Food and Agriculture Organization (FAO) (2020) The State of World Fisheries and Aquaculture, In brief.

7. Galagarza OA, Smith SA, Drahos DJ, Eifert JD, Williams RC, Kuhn DD (2018) Modulation of innate immunity in Nile tilapia (Oreochromis niloticus) by dietary supplementation of Bacillus subtilis endospores. Fish Shellfish Immunol 83:171-179

8. Guendouze A, Plener L, Bzdrenga J, Jacquet P, Rémy B, Elias $M$, Lavigne JP, Daudé D, Chabrière $E$ (2017) Effect of quorum quenching lactonase in clinical isolates of Pseudomonas aeruginosa and comparison with quorum sensing inhibitors. Front Microbiol 8:227 
9. Gui L, Zhang QY (2018) Disease Prevention and Control. in: Gui JF, Tang Q, Li Z, Liu J, De Silva SS (ed) Aquaculture in China: Success Stories and Modern Trends. Wiley, London, England, pp. 577-598

10. Zhang G, An Y, Hossain Z, Guo Q, Yang M, Yuan J, Wen L, Sun W, Qi X (2019) Bacillus subtilis spore surface display technology: A review of its development and applications. J Microbiol Biotechn 29(2):179-190

11. Haque S, Ahmad F, Dar SA, Jawed A, Mandal RK, Wahid M, Lohani M, Khan S, Singh V, Akhter N (2018) Developments in strategies for quorum sensing virulence factor inhibition to combat bacterial drug resistance. Microb Pathogenesis 121:293-302

12. He S, Ran C, Qin C, Li S, Zhang H, de Vos WM, Ringo E, Zhou Z (2017) Anti-infective effect of adhesive probiotic Lactobacillus in fsh is correlated with their spatial distribution in the intestinal tissue. Sci Rep 7:13195

13. Høyland-Kroghsbo NM, Paczkowski J, Mukherjee S, Broniewski J, Westra E, Bondy-Denomy J, Bassler BL (2017) Quorum sensing controls the Pseudomonas aeruginosa CRISPR-Cas adaptive immune system. Proc Natl Acad Sci U S A 114(1):131-135

14. Huang T, Song XH, Zhao KL, Jing J, Shen YM, Zhang XY, Yue BS (2018) Quorum-sensing molecules $\mathrm{N}$-acyl homoserine lactones inhibit Trueperella pyogenes infection in mouse model. Vet Microbiol 213:89-94

15. James G, Das BC, Jose S, Kumar RVJ (2021) Bacillus as an aquaculture friendly microbe. Aquacult Int 29(1):323-353

16. Jang WJ, Hasan MT, Lee GH, Lee BJ, Hur SW, Lee S, Kim KW, Lee EW, Kong IS (2021) Comparison of spore or vegetative Bacillus sp. supplementation on physiological changes and gut microbiota of the olive flounder (Paralichthys olivaceus). Aquaculture 535:736355

17. Jiang Q, Chen J, Yang C, Yin Y, Yao K (2019) Quorum sensing: A prospective therapeutic target for bacterial diseases. BioMed Res Int 2019:2015978

18. Kalia VC, Patel SKS, Kang YC, Lee JK (2019) Quorum sensing inhibitors as antipathogens: biotechnological applications. Biotechnol Adv 37(1):68-90

19. Kariminik A, Baseri-Salehi M, Kheirkhah B (2017) Pseudomonas aeruginosa quorum sensing modulates immune responses: An updated review article. Immunol Lett 190:1-6

20. La Ragione RM, Woodward MJ (2003) Competitive exclusion by Bacillus subtilis spores of Salmonella enterica serotype Enteritidis and Clostridium perfringens in young chickens. Vet Microbiol 94(3):245-256

21. Latorre JD, Hernandez-Velasco X, Kallapura G, Menconi A, Pumford NR, Morgan M.J, Layton SL, Bielke LR, Hargis BM, Tellez G (2014) Evaluation of germination, distribution, and persistence of Bacillus subtilis spores through the gastrointestinal tract of chickens. Poultry Sci 93(7):1793-1800

22. Lieke T, Meinelt T, Hoseinifar SH, Pan B, Straus DL, Steinberg CEW (2019) Sustainable aquaculture requires environmental-friendly treatment strategies for fish diseases. Rev Aquacult 12(2):943-965

23. Liu Z, Liu W, Ran C, Hu J, Zhou Z (2016) Abrupt suspension of probiotics administration may increase host pathogen susceptibility by inducing gut dysbiosis. Sci Rep 6:23214 
24. Mion S, Remy B, Plener L, Bregeon F, Chabriere E, Deude D (2019) Quorum quenching lactonase strengthens bacteriophage and antibiotic arsenal against Pseudomonas aeruginosa clinical isolates. Front Microbiol 10:2049

25. Mukherjee S, Bassler BL (2019) Bacterial quorum sensing in complex and dynamically changing environments. Nat Rev Microbiol 17(6):371-382

26. Nayak S (2021) Multifaceted applications of probiotic Bacillus species in aquaculture with special reference to Bacillus subtilis. Rev Aquacult 13(3):862-906

27. Naylor RL, Hardy RW, Buschmann AH, Bush SR, Cao L, Klinger DH, Little DC, Lubchenco J, Shumway SE, Troell M (2021) A 20-year retrospective review of global aquaculture. Nature 591(7851):551-563

28. Novoa B, Bowman TV, Zon L, Figueras A (2009) LPS response and tolerance in the zebrafish (Danio rerio). Fish Shellfish Immunol 26:326-331

29. Olmos J, Acosta M, Mendoza G, Pitones V (2020) Bacillus subtilis, an ideal probiotic bacterium to shrimp and fish aquaculture that increase feed digestibility, prevent microbial diseases, and avoid water pollution. Arch Microbiol 202(3):427-435

30. Oyarbide U, Iturria I, Rainieri S, Pardo MA (2015) Use of gnotobiotic zebrafish to study Vibrio anguillarum pathogenicity. Zebrafish 12(1):71-80

31. Pan X, Yang Y, Liu X, Li D, Li J, Guo X, Zhou Z (2016) Secretory expression of a heterologous protein, Aiio-AlO6BS, in Bacillus subtilis via a non-classical secretion pathway. Biochem Bioph Res Co 478(2):881-886

32. Papenfort K, Bassler BL (2016) Quorum sensing signal-response systems in Gram-negative bacteria. Nat Rev Microbiol 14(9):576-588

33. Ran C, Qin C, Xie M, Zhang J, Li J, Xie Y, Wang Y, Li S, Liu L, Fu X, Lin Q, Li N, Liles MR, Zhou Z (2018) Aeromonas veronii and aerolysin are important for the pathogenesis of motile aeromonad septicemia in cyprinid fish. Environ Microbiol 20(9):3442-3456

34. Shi F, Zi Y, Lu Z, Li F, Yang M, Zhan F, Li Y, Li J, Zhao L, Lin L, Qin Z (2020) Bacillus subtilis H2 modulates immune response, fat metabolism and bacterial flora in the gut of grass carp (Ctenopharyngodon idellus). Fish Shellfish Immunol 106: 8-20

35. Soltani M, Ghosh K, Hoseinifar SH, Kumar V, Lymbery AJ, Roy S, Ringø E (2019) Genus bacillus, promising probiotics in aquaculture: Aquatic animal origin, bio-active components, bioremediation and efficacy in fish and shellfish. Rev Fish Sci Aquac 27(3):331-379

36. Subedi B, Shrestha A (2020) A review: Application of probiotics in aquaculture. Int J Forest, Animal Fisheries Res 4(5):52-60. DOI: 10.22161/ijfaf.4.5.1

37. Suva M, Sureja V, Kheni D (2016) Novel insight on probiotic Bacillus subtilis: Mechanism of action and clinical applications. J Current Res Sci Med 2(2):65-72

38. Tan F, Limbu SM, Qian Y, Qiao F, Du ZY, Zhang M (2019) The responses of germ-free zebrafish (Danio rerio) to varying bacterial concentrations, colonization tme points, and exposure duration. Front Microbiol 10:2156 
39. Teame T, Wu X, Hao Q, Ding Q, Liu H, Ran C, Yang Y, Xia L, Wei S, Zhou Z, Zhang Y, Zhang Z (2020) Dietary SWF ${ }^{\circledR}$ enhanced growth performance and disease resistance in hybrid sturgeon (Acipenser baerii x Acipenser schrenckii) mediated by the gut microbiota. Aquacult Rep 17(4):100346

40. Wong SL (1995) Advances in the use of Bacillus subtilis for the expression and secretion of heterologous proteins. Curr Opin Biotech 6(5):517-522

41. Wu X, Teame T, Hao Q, Ding Q, Liu H, Ran C, Yang Y, Zhang Y, Zhou Z, Duan M, Zhang Z (2020a) Use of a paraprobiotic and postbiotic feed supplement (HWF) improves the growth performance, composition and function of gut microbiota in hybrid sturgeon (Acipenser baerii x Acipenser schrenckii). Fish Shellfish Immunol 104:36-45

42. Wu X, Hao Q, Teame T, Ding Q, Liu H, Ran C, Yang Y, Xia L, Wei S, Zhou Z, Zhang Z, Zhang Y (2020b) Gut microbiota induced by dietary $\mathrm{GWF} \circledast$ contributes to growth promotion, immune regulation and disease resistance in hybrid sturgeon (Acipenserbaerii x Acipenserschrenckii): Insights from a germfree zebrafish model. Aquaculture 520:734966

43. Xia R, Yang Y, Pan X, Gao C, Yao Y, Liu X, Teame T, Zhang F, Hu J, Ran C, Zhang Z, Liu-Clarke J, Zhou $Z$ (2020) Improving the production of AHL lactonase AiiO-AlO6 from Ochrobactrum sp. M231 in intracellular protease-deficient Bacillus subtilis. AMB Expr 10:138

44. Yao YY, Yang YL, Gao CC, Zhang FL, Xia R, Li D, Hu J, Ran C, Zhang Z, Liu-Clarke J, Zhou ZG (2020) Surface display system for probiotics and its application in aquaculture. Rev Aquacult 12(4):23332350

45. Yu Y, Wang Q, Huang Z, Ding L, Xu Z (2020) Immunoglobulins, mucosal immunity and vaccination in teleost fish. Front Immunol 11(2597):567941

46. Zhang M, Cao Y, Yao B, Bai D, Zhou Z (2011) Characteristics of quenching enzyme AiiO-AlO6 and its effect on Aeromonas hydrophila virulence factors expression. J Fisheries China 35(11):1720-1728. https://www.cabdirect.org/cabdirect/abstract/20123070785

47. Zhou C, Wang H, Li X, Luo Y, Xie M, Wu Z, Chen X (2019) Regulatory effect of Bacillus subtilis on cytokines of dendritic cells in grass carp (Ctenopharyngodon Idella). Int J Mol Sci 20(2): 389

\section{Tables}

Table 1 Primers used in this study. 


\begin{tabular}{|c|c|c|c|}
\hline Gene & Forward primer $\left(5^{\prime}-3^{\prime}\right)$ & Reverse primer $\left(5^{\prime}-3^{\prime}\right)$ & Application \\
\hline AiiO-AIO6 & AAATCCCATGAAATCGAGACCA & GCCGTGCAGTCGCGCATGA & \multirow{2}{*}{$\begin{array}{l}\text { genomic PCR } \\
\text { detection }\end{array}$} \\
\hline amyE & $\begin{array}{l}\text { ATGTTTGCAAAACGATTCAAAA } \\
\text { CC }\end{array}$ & TCAATGGGGAAGAGAACCGCT & \\
\hline Rps11 & ACAGAAATGCCCСTTCACTG & GCСTCTTCTCAAAACGGTTG & \multirow[t]{6}{*}{ qPCR } \\
\hline $\mathrm{IL}-1 \beta$ & GGCTGTGTGTTTGGGAATCT & TGATAAACCAACCGGGACA & \\
\hline NF-KB & AGACACCGACAGCAGTCAGGAG & GGTGACGGAGGTTGAAGAAGGATG & \\
\hline IL-10 & ATTTGTGGAGGGCTTTCCTT & AGAGCTGTTGGCAGAATGGT & \\
\hline TGF- $\beta$ & GTGTACCCGCAATCCTTGAC & САCTTGCAGTTCCTCACCAC & \\
\hline lysozyme & TGGAAGTGGTGTTTTTGTGT & TCAAATCCATCAAGCCСТTC & \\
\hline
\end{tabular}

Table 2. Count of $A$. veronii Hm091 cocultured with B. subtilis wt55 or AiiO-AlO6.

If the two groups do not contain the same letter, the difference is significant $(p<0.05)$, and the same letter appearing means no significant change. Data are means for three

\begin{tabular}{|c|c|c|c|c|}
\hline & ck & AiiO-AlO6 & wt55+Al06 & wt55 \\
\hline $4 \mathrm{~h}$ & $2.05 \pm 0.33 * 10^{9} \mathrm{a}$ & $2.42 \pm 0.39 * 10^{9} \mathrm{a}$ & $2.16 \pm 0.86 * 10^{9} \mathrm{a}$ & $1.73 \pm 0.57 * 10^{8 b}$ \\
\hline $8 \mathrm{~h}$ & $5.54 \pm 1.78 * 10^{10 a}$ & $5.54 \pm 0.33^{*} 10^{10 a}$ & $2.37 \pm 0.63 * 10^{10 b}$ & $2.71 \pm 0.48 * 10^{10 b}$ \\
\hline $12 \mathrm{~h}$ & $3.71 \pm 1.07 * 10^{11 \mathrm{a}}$ & $4.23+1.57 * 10^{11 \mathrm{a}}$ & $2.52 \pm 0.90 * 10^{11 a}$ & $4.37 \pm 1.58 * 10^{11} a$ \\
\hline
\end{tabular}

Table 3. Strains and plasmids used in this work. 


\section{Strains and Characteristics}

plasmids

Strains

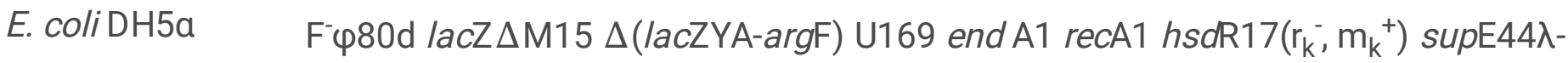
thi-1 gyrA96 relA1 phoA

A. Peronii Hm091 Pathogen, $\mathrm{Amp}^{\mathrm{r}}$
ver

B. subtilis wt55 Probiotics, isolated from sturgeon

wt55-N-AlO6 $\mathrm{Cm}^{\mathrm{r}}$, AiiO-AlO6 could only surface displayed on the spores of wt55

(SD)

wt55-AlO6 $\mathrm{Cm}^{\mathrm{r}}$ and $\mathrm{Kan}^{\mathrm{r}}$, AiiO-AlO6 could surface displayed on the spores of wt55 and secreted by its vegetative cells

Plasmids

pDG364-N-AIO6 $\mathrm{Cm}^{\mathrm{r}}$, replicate in E. coli, homologous recombination in B. subtilis at amylase gene

pWB-AIO6BS Kan ${ }^{r}$, only replicate in B. subtilis, constitutive secretory expression of protein

Amp: Ampicillin; Cm: Chloramphenicol; Kan: Kanamycin; r: resistance.

\section{Figures}


a

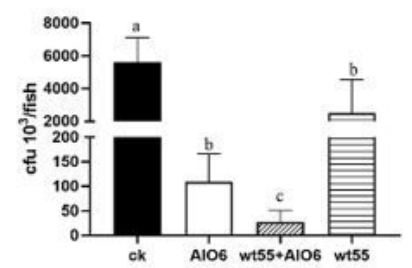

b

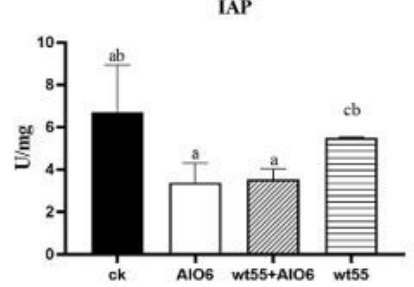

$\mathrm{c}$

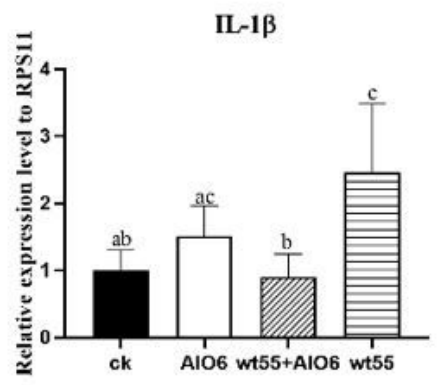

$\mathrm{d}$

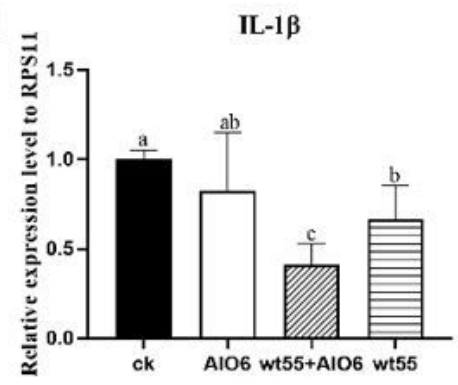

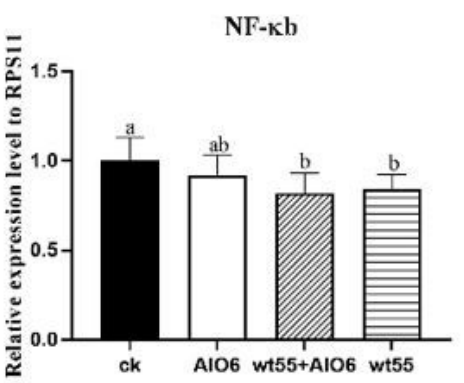
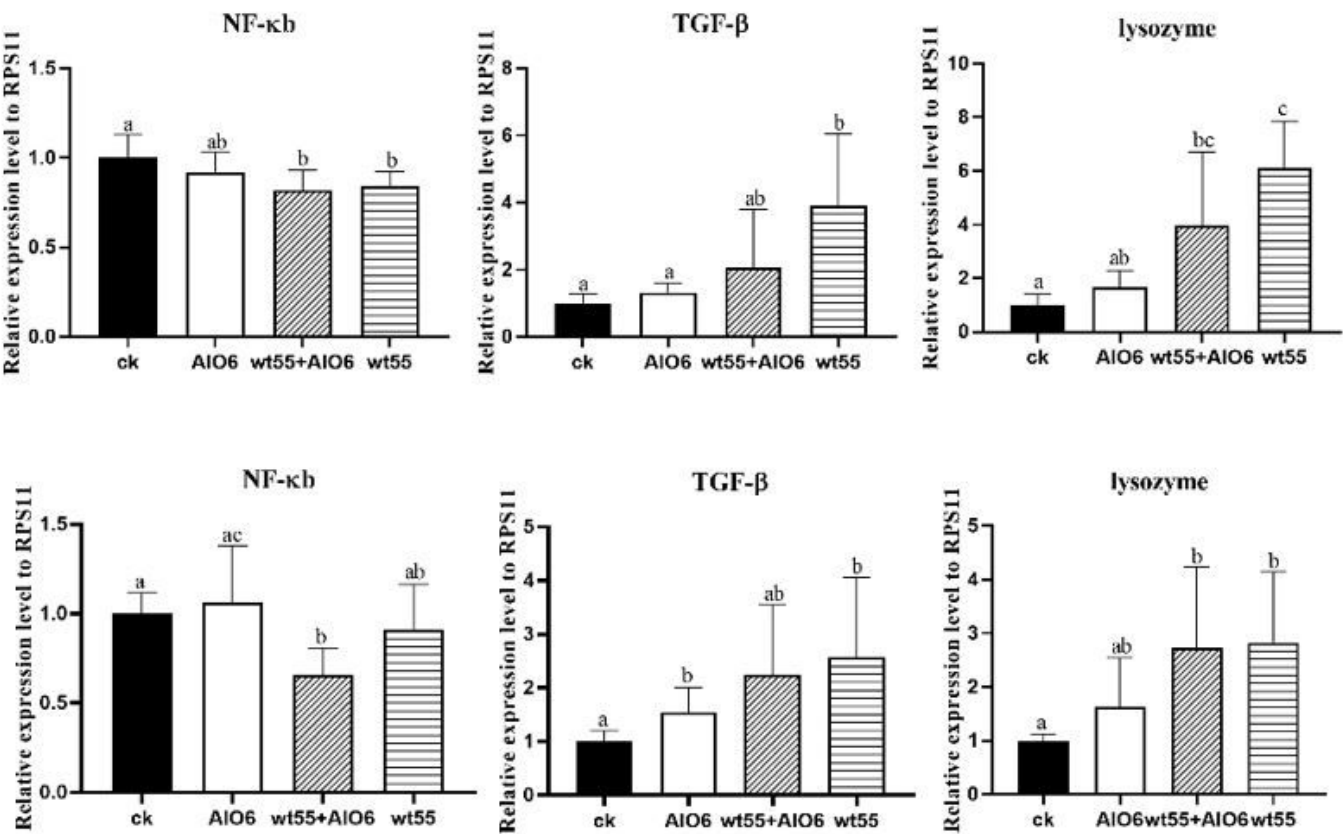

Figure 1

Effect of B. subtilis combinate with AiiO-AIO6 on disease resistance of zebrafish. a Abundance of A. veronii $\mathrm{Hm} 091$ in the gut of zebrafish $24 \mathrm{~h}$ after challenge. b IAP activity of zebrafish $24 \mathrm{~h}$ after challenge with A. veronii Hm091. c The expression of non-specific immune related genes of different formulations after directly immerse of GF zebrafish model. d Effect of gut microbiota induced by different formulations on the expression of non-specific immune related genes in the GF zebrafish model. If the two groups do not contain the same letter, the difference is significant $(p<0.05)$. The same letter appearing means no significant change. Data are means for six replicates and presented as means \pm SD. 


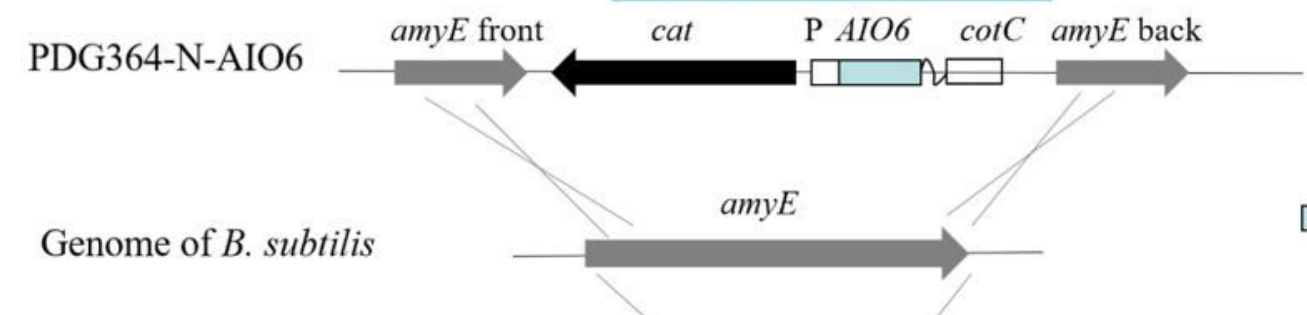

Homologous recombination

B. Subtilis wt55-AIO6 strain
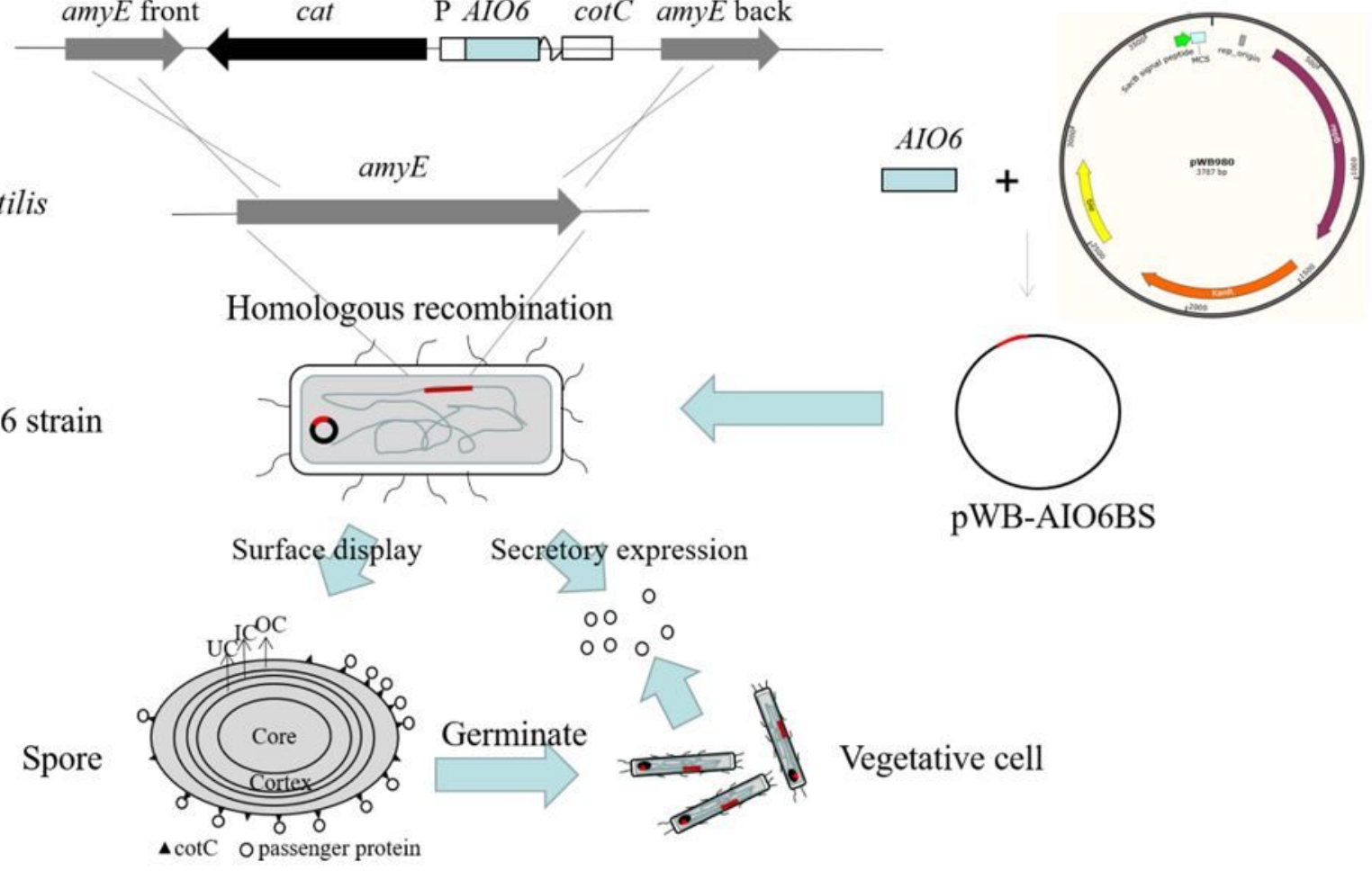

\section{Figure 2}

Schematic view of building quorum-quenching recombinant strain wt55-Al06. Abbreviations: cat, Chloramphenicol resistance gene; P, promoter; UC, undercoat; IC, inner coat; OC, outer coat. 
a
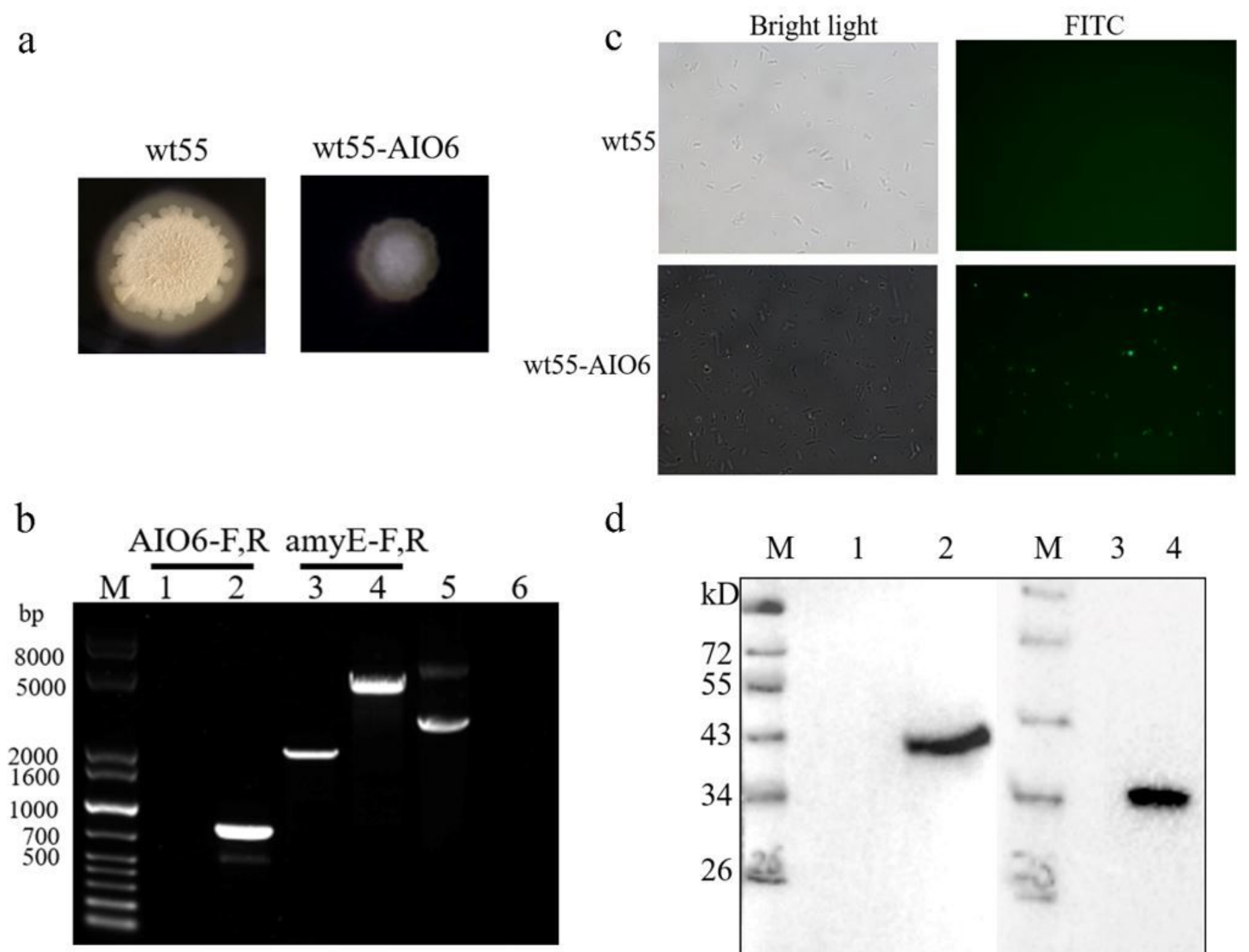

d

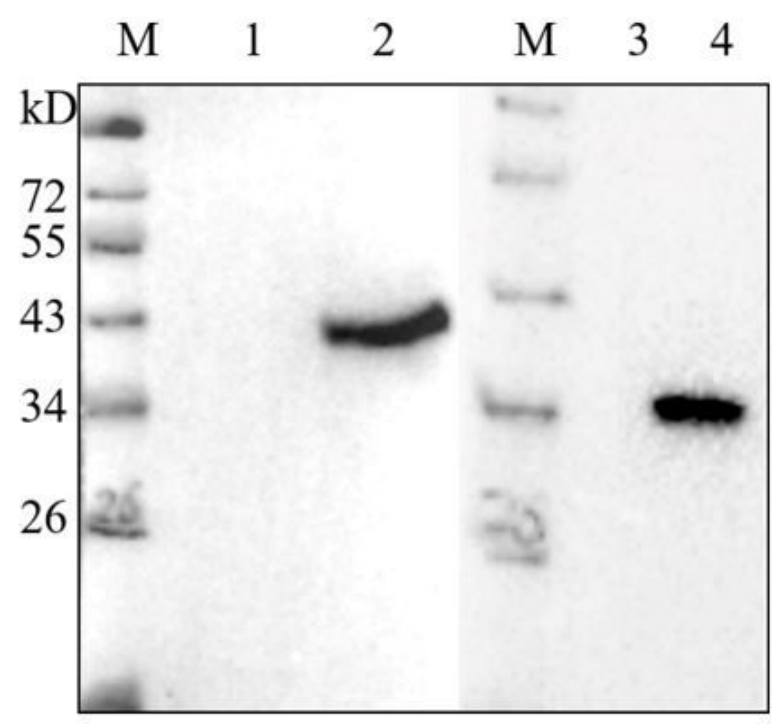

\section{Figure 3}

Validation of QQ recombinant wt55-AlO6 strain included surface display and secretory expression. a Analysis of amylase activity. B. subtilis wt55 and wt55-Al06 recombinant strains on starch-containing LB plate stained by iodine. b PCR analysis of B. subtilis genome, primer pairs used are labelled above. And plasmid extraction and identification. Line 1, 3 and 6: wt55, line 2, 4 and 5: wt55-AIO6. C Immunofluorescent detection of recombinant spores. $\mathrm{d}$ WB analysis of proteins extracted from spores and AiiO-AlO6 secreted into medium. The target protein was detected by anti-His antibody. Line 1 and 3 : wt55, line 2 and 4: wt55-AIO6. 
a

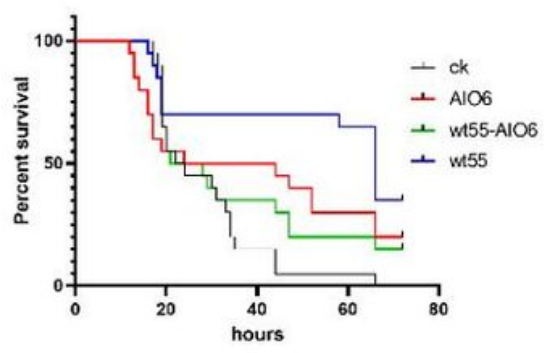

b

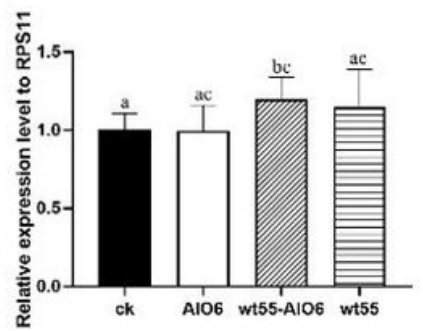

NF-кB

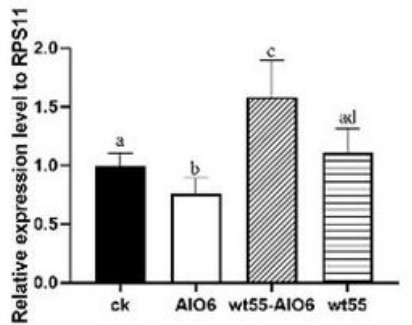

IL-10

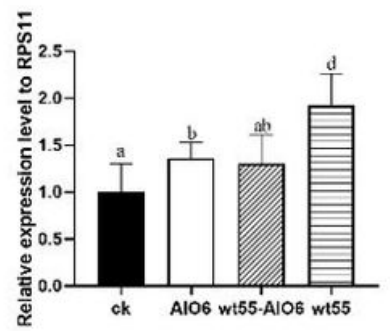

Figure 4

Disease resistance of zebrafish fed with recombinant B. subtilis wt55-Al06 strain. a The survival curves of zebrafish feed with native wt55 spores or recombinant wt55-Al06 spores or AiiO-Al06 after challenged by A. veronii, and basal diet (ck) as control. b qRT-PCR analysis of expression of inflammatory responserelated genes in zebrafish dieted with different formulations in gut after breeding experiment. If the two groups do not contain the same letter, the difference is significant $(p<0.05)$. The same letter appearing means no significant change. Data are means for six replicates and presented as means \pm SD. 